\title{
Annals of Cognitive Science
}

ISSN: $2642-4290$

Review Article

DOI: $10.36959 / 447 / 349$

\section{Central Nervous System Model Theory- A Universal Model of all Neural Processes}

\author{
Maha Osman Mohamed Shangab* \\ Internal Medicine Department, Rashid Hospital, Dubai Health Authority, Dubai, UAE
}

\begin{abstract}
To a first time examiner, our brains capabilities and functions would seem to differ between individuals. No two men are alike. Taking a step back, however, and examining the human population, one can detect the similar traits present in all the seemingly different brain functions. These similarities include four characteristics, which are:

1) Subconscious operation

2) Automatism

3) Innate predisposition and neural plasticity

4) Frameworks for practical operation that are both generalizable and flexible to allow practical application

The theoretical hypothesis advanced in this article provides explanation for these characteristics as well as a collection of evidence to support their notion.
\end{abstract}

\section{Keywords}

Memory, Perception, Decision-making, Subconscious, Automaticity

\section{Introduction}

The mind is a realm of great wonder. It's the last frontier of scientific investigations. What we know about it so far is minute compared to what we believe remains in the unknown. However, it is not necessary to have a comprehensive idea regarding its mechanistics to understand the principles that governs its functions. Similar to newton's three laws of motion; there are laws that govern the neural processes that manifest as the mind, perception and behavior.

In the upcoming paragraphs, I attempt to propose a unified model whose characteristics can explain the principles by which the neural models develop and function. What is discussed here should not come as a new discovery as most of the evidence provided for the theoretical framework comes from an already discovered data. The framework for the theory is rather a set of principles that all neural processes have in common and operate by. The models mentioned involve all central nervous system neural processes, hence the term universal.

I advance the notion that neural processes are based on models. These models are to a great limit constant and similar among humans because they share similar processes for operation. Box 1 summarizes the proposed principles that unify such models.

\section{Box 1}

The common characteristics between all the models in the nervous system include:

1) They are subconsciously operated.

2) They are mostly automated. The less conscious they are, the more automated their function is.

3) The predisposition for them is sat innate but undergoes fine tuning through learned adaptive experiences "innate dispositions and neural plasticity".

4) They are not situation specific but rather a group of generalizable laws and programs of action that have both the flexibility and the ability to be applied in relevant situations.

*Corresponding author: Maha Osman Mohamed Shangab, MD, Internal Medicine Department, Rashid Hospital, Dubai Health Authority, Umhurair, Dubai, UAE, Tel: 00971504400152

Accepted: September 09, 2020

Published online: September 11, 2020

Citation: Shangab MOM (2020) Central Nervous System Model Theory- A Universal Model of all Neural Processes. Ann Cogn Sci 4(1):166-173

Copyright: (C) 2020 Shangab MOM. This is an open-access article distributed under the terms of the Creative Commons Attribution License, which permits unrestricted use, distribution, and reproduction in any medium, provided the original author and source are credited. 


\section{Introduction into the theory}

Every neural process in our mind has a pre-sat default setting for operation. This encompasses processes that range from the simplest withdrawal reflex to the fight and flight reaction to something as specific as watching a movie or reading a book or as personal as deciding on a hobby.

All neural functions are not fully experience influenced. This comes as a common knowledge for basic functions like reflexes. But the reality of it encompasses all functions, such as perception, memory and individual decision-making.

Degree of flexibility and rigidity of the system function largely depends on its location between the unconscious conscious spectrum. The more conscious the thought, percept or act is, the more flexible or rather modifiable the process of it can be; the less conscious the neural process, the more automated is the influence of the default system on it. However, this should be taken with a grain of salt, as even the most conscious of actions such as decision making operates on a default system and is to a large degree automated, evidence of such notion is presented later in text as the somatic marker hypothesis proposed by Antonio Damasio [1].

Whatever runs consciously in the mind, runs in models of operation that are first laid down by innate systems. The innate system is based on genes expression which is then refined by experience. The degree of refinement by experience, or "plasticity", is most effective during early periods of development in most brain regions [2]. Neuroplasticity remains to a certain degree during adulthood, but is more pronounced in certain areas than others. It should be noted, however, that even with that greatest levels of plasticity, there is a constant frame for development that continuous [2].

The hippocampus is an example of a sector that retains its plasticity and ability to generate new cells for most of the life span as this ability serves its function of memory consolidation. Its decline is usually associated with memory impairment seen in aging or dementias [3]. This ability to retain neuroplasticity is likely the result of neural stem cells located in the subgranular zones in the dentate gyrus [3].

Despite our understanding for several neural pathways in the central nervous system, a comprehensive picture was lacking. This is the gap this article attempts to fill.

\section{Principles of Neural Models}

I propose that all central neural processes are based on models. These models are to a great limit constant and similar among humans. The common characteristics between all the models in the nervous system include:

1) They are subconsciously operated.

2) They are mostly automated. The less conscious they are, the more automated their function is.

3) The predisposition for them is sat innate but undergoes fine tuning through learned adaptive experiences "innate dispositions and neural plasticity".

4) They are not situation specific but rather a group of gen- eralizable laws and programs of action that have both the flexibility and the ability to be applied in relevant situations.

\section{Subconscious operation}

The subconscious is not a new term, nor is it a recent concept. Even before Freud has introduced its applicability in psychoanalysis, the subconscious has been a matter of practice and debate.

Poetically, subconscious neural processes can be described as being beyond the realm of conscious processes, rather hiding behind the curtain and pulling their controlling threads. In reality, however, it's the way our neural faculties function according to their default programing. Consciousness is in fact, an additional mode of operation that allows us to be aware about some of the neural processes. What it doesn't allow us to be aware about, we term subconscious. Additionally, consciousness is not embodied in a center in the brain, but rather the result of a network of mechanisms that result in the organism being aware and 'conscious' about itself [4].

\section{Automation}

Automaticity according to oxford definition is the ability to perform a task automatic processing, independent of conscious control and attention. Following this definition, it comes clear that some neural processes are automatic, such as the simple knee reflex and other reflexes that have been studied extensively.

There is, however, a degree of automation that exists in more complicated processes as well. Taking motor activities for example, one can drive a car once learned, without the same level of conscious effort at achieving movements' coordination. This comes after the behavior is consciously finetuned by associate motor cortex circuits with the basal ganglia and after the circuit becomes modified by motor memory consolidation. Perception follows the same principle, reading becomes a fluent process once we accumulate the phonological and semantic data related to it and retrieve it and integrate it during our perception of the written text [5].

Rapid automatized naming is usually used as a measuring tool as an index of automaticity in reading. It's a test that requires naming common objects like letters and colors as fast as possible [5]. The test is associated with the activation of the following sites that are termed as 'reading network'": Frontal cortex, left-hemisphere dorsal posterior regions and ventral visual pathway [6].

Automation is the rule in all neural circuits. It doesn't require our full awareness and conscious control to operate. This even includes decision making. Part of its automation comes from the fact that decisions are made in a subconscious level before conscious awareness takes place. Surprisingly, this can also occur after deliberate conscious thought process, but not always. This is aimed at helping thought process to be more time efficient as well as wiser at decision making, at least in term of making decisions that support survival [1].

I do not wish to imply here that we are passive observers 
by mentioning how our neural processes are automated. But imagine a person who is awake but inattentive. He would go about his work, drive home, eat and sleep. Attention not only allows us to be more aware about what we see, think and do, but it gives us the power to modify our thinking processes and thus our actions. Consciousness allows us to deliberately think and evolve as social humans.

\section{Innate disposition and developmental plasticity}

How our brains should develop and the possible potentials for that development are already coded in our genes. Their gene expression is sequenced in a manner that allows them to develop in the form they currently exist in. Despite the presence of minor anatomical variations in the neuroanatomy of our nervous system, the general scheme remains the same.

The same principle is reflected in microscopic and molecular scales. There are innate dispositions for the functional arrangement of neural circuits in our nervous system. These innate dispositions do develop as our mind develops, but the limit for such development depends on the developmental capacity and adaptability of each specific sector. This developmental capacity and adaptability is termed plasticity. Neural plasticity can be broadly defined as our nervous system ability to change and adapt in response to stimuli, wither internal or external [7]. Neural plasticity is the capacity to be shaped by experience, to adapt to new physiological, as well as pathological conditions and stimuli.

The function and arrangement of most neural processes such as perception, is determined by genes expression. There is, however, a degree of fine tuning or reforming that occurs with development and through experience. This specifically applies to learned processes, higher cortical functions and subcortical functions such as memory, motor circuits, skills and decision making.

\section{Models are frameworks for practical application}

The proposed models of operation are not situation specific but rather a group of generalizable laws and programs of action that have both the flexibility and the ability to be applied in relevant situations. This ability enables cost effectiveness of brain circuits. If that was not the case, our brains would have to be much larger to accommodate all its functions. This rule is applied in all bodily organs.

The purpose here is not solely for cost effectiveness of Circuits in terms of volume and complexity only, but to allow us to develop in our environment. Imagine being tasked to do a repetitive job like writing invitation letters in a short time frame. You would simply develop a template and make copies of it with different names on top. The invitation template is generalizable and the names are specific. This example demonstrates how we, as well as our brain, always function to create systems that allow us to work more efficiently.

Our theory expands Peiage notion of assimilation and accommodation of knowledge [8] assimilation denotes according to him the interpretation and acquisition of new information in terms of pre-existing concepts, information or ideas, and accommodation means the revision and adjustment of previous knowledge and concepts in terms of the new experiences. Both processes occur together to achieve cognitive equilibrium. The principle of cognitive equilibrium doesn't apply to knowledge alone or schemata as he initially suggested, but also to executive actions such as improvement of procedural skills and decision making, as well as perceptive skills [9].

\section{Evidence}

The abovementioned characteristics are known and accepted regarding the simple models or 'reflexes' and 'circuits' such as knee reflex and fight and flight response present in the peripheral nervous system. For this reason I will limit my arguments to the processes present in the central nervous system that appear more individual specific, private and for a long time have fallen out of the scope of science and into the realm of psychology. Processes that would be discussed include:
1) Perception
2) Memory consolidation and retrieval
3) Decision making

\section{Perception}

Perception follows generalizable laws and models of operation: When it comes to perception, the input of any stimulus into the nervous system being interoceptive, proprioceptive or exteroceptive follows to a certain extent, the same mechanism to form a mental image of that percept. To believe perception is a simple recording of events is over simplification to a fault of the actual mechanism occurring in the brain.

Perception is far from the simplistic idea of capturing an image like a camera and storing it in a shelf. Perception is actually a breaking down of different components of perception then transmitting those impulses to the cortex. The cortex 'actively' constructs the images in the brain. It builds the mental image of a vision, sound, taste or touch by using previously formed models of representations of perception and fitting the details received by the sensory receptors into it.

For the purpose of illustration, we'll describe the process of visual perception. Ancient thoughts believed light emanated from eyes and allowed vision. Later on discoveries of optics and light waves allowed our understanding of how the retina perceives light frequencies and visual images are formed.

When a light enters the eyes, it passes through the cornea, lens and vitreous fluid to land in the retina. There, the photoreceptors - rods and cones - are stimulated according to the light frequency and intensity. From that point, the neural visual imagery starts. The action potential impulses get transmitted through the visual tract starting with the optic nerve, optic tract, and optic radiation till it reaches the occipital cortex, specifically, the primary visual cortices, V1. There are other collateral tracts that leave from the lateral geniculate body to the superior colliculus and Edinger-Westphal nuclei, but those are outside our current scope [10]. 
Once the visual impulse reaches the primary visual cortex, the picture starts building up. From that point an anterior impulse transmission occurs towards the temporal lobe. Initially basic features like orientation are deciphered, followed by more complex details such as shape, edge, contrast. Finally the image is integrated with semantic data [11]. This continuous with a backward feedback to fine tune the percept impulse, wither according to the focus of attention or other reasons. A continuous forward, backward loop continues to better enhance visual perception according to what is most relevant in reference to the information needed and the focus of attention [11].

Visual imagery or the ability to retrieve a memory with visual details follows anterior to posterior flow from the fronto-parietal areas backwards to temporal and occipital areas [12]. This can explain why when we remember visual scenes, we remember the semantic relations it holds to us first then the perceptual details it holds.

This model of perception and construction of visual scenes in the brain processes information received from the retina by following generalizable laws that are based on our knowledge of how the visual world should appear to us. We don't experience reality as it exists; we experience it as our mind builds it up for us. For their purpose of efficiency, they simply fill details about what we see in the most relevant visual representation frame it has. They also give corrective feedback regarding how visual perception should be processed by omitting the blind spot from the visual perception or bring about visual illusions for example.

Adelson's Checker-Shadow illusion is a famous example of that [13]. The image demonstrates a checkerboard with white and dark squares. When a shadow is casted on a board, we expect the pattern to continue as it is and expect the object to darken the areas under the shadow. This is where the optical illusion lies; we mistakenly misperceive the degree of grayness in order to fit it in the model of representations that is preformed in our brain circuits (Figure 1).

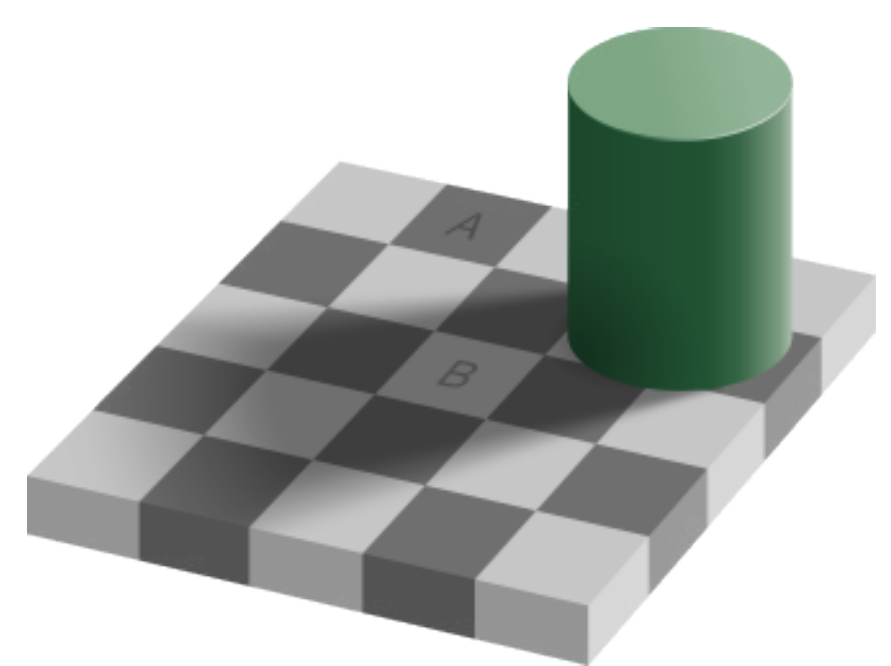

Our perception of the outside world (exteroception), our place in it (prioprioception) and our insides (interception) is an act of recording specific details about it and building up the remaining details into a preformed framework about how that modality should be experienced in our mind.

Receptors are designed to register only a specific modality of stimulation. An example of that is seeing flashes of light when the rods get stimulated mechanically during vitreous detachment. There are no real flashes, yet this is what our mind builds for us.

Perception mechanism is innately formed but undergoes a level of plasticity as we mature: Perception is innately developed; however, it undergoes extensive modification with early perception and discovery of the world during infant and toddler life. By the time we become adults, our store of perception concepts that build the perception models become extensive. These models are framed to agree with known knowledge about how the universe should be experienced to us. When perception contradicts these preconceived concepts about reality, an illusion develops. The more we develop these models, the more reliant we become on them. With time, our ability to generate more models reduce and 'plasticity reduces'.

Plasticity is seen, to a limit, throughout life as recent evidence suggest, but not in all regions [2]. The level of plasticity is greatest at childhood and it lessens with age. How modifiable our neural circuits lessen with age. But even with the greatest level of plasticity, there is a constant frame for development that any possible modification and change is bound to follow and be constrained with. Otherwise, reality would never be the same for everyone.

Neural plasticity can include changes in the cellular level by affecting synaptic connections, or systemically by causing structural changes in cortico-cortical and cortico-subcortical pathways, or functional plasticity by modifying the mapping between behavior and neural activity [14].

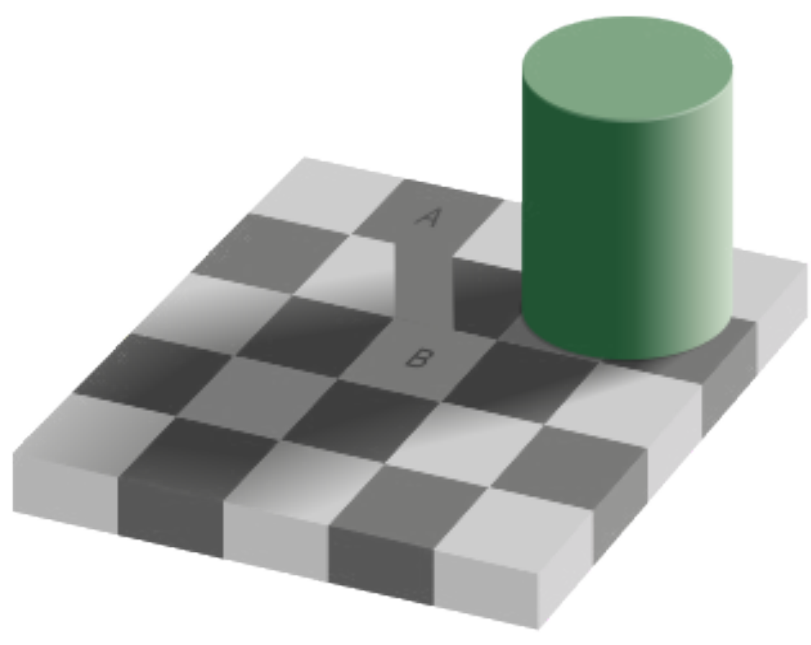

Figure 1: Adelson's checker-shadow illusion.

([14] Free Copyright). 
We rely on the fact that reality is a relatively constant term between individuals. We believe that what we see, what we hear, and how we can possibly react in certain situations is similar among all our species. This is how we connect, and this is how we relate to one another and how we empathize.

Plasticity also allows people to adapt to experiential events. When a sector of the brain sustains an injury, healing takes place. Plasticity allows the brain to heal in a way to allow functionality to persist sometimes in the same form, and sometimes in different form.

Taking again the example of visual perception, after sustaining an injury in any area of the visual cortex, the outcome lies somewhere between the complete equipotentiality theory where any other part of the cortex will take place and functional recovery will occur $100 \%$ and the fixed localization of functions where absent recover would ensue. A flexible and dynamic, experience-dependent outcome usually results [14].

A review of 46 pediatric cases did not reveal a pattern that can indicate potential factors that influence recovery and mode of plasticity [14], but they were case reports that failed to report the personal aspect of the experiences. Oliver sacks in his book mind's eye gave report about several cases who lost their vision at different points in life [15]. What seemed to guide the mode of recovery in each case experienced was first, the age at which the lesion developed, and second, how the individual reacted to such loss. Two people who lost their vision during adulthood had two different recoveries. One has focused on his visual imagery and with time, this enhanced his visual function still he was able to build mental representations regarding his environment with ease. Another has forgotten his visual imagery, or rather surrendered to darkness and with time, have gained enhancement of other senses that he became able to experience the world through them. A person who was congenitally blind was also interviewed; he was unable to understand how he was blind, as visual imagery did not hold a meaning to him as it did to us. There was no darkness, but absence of the idea of 'seeing' [15].

Perception is automated and subconsciously operated: We don't have to be conscious of our world to experience it. It may seem counter intuitive, but the mechanism of perception doesn't rely on conscious processing to operate. Studies from several cases, including a study on healthy adults regarding unconscious visual perception showed that at values of $50 \mathrm{~ms}$ of visual stimulation, subjects reported no awareness but were able to answer with a great degree of accuracy in a nonrandom fashion indicating the presence of subconscious visual processing [16]. A meta-analysis involving functional MRI testing of conscious and subconscious visual processing showed activation of right fusiform gyrus, right caudal anterior cingulate cortex and right insula during unconscious visual perception, with activation of left rostral anterior cingulate cortex in conscious perception [17].

Perception also runs automatically. Studies in cases with anxiety disorder revealed that perception of a threat occurs involuntarily and fast. When a threat is present in visual vicinity, there is an element of attention bias that guides the or- ganism to identify the threat quickly to allow rapid response [18].

\section{Memory consolidation and retrieval}

Memory follows perception in order of activity. What we perceive we record. Similar to the simplistic idea we seem to like for perception, we do the same for memory. We think of memory as a simple video recording of perceptive events that can be replayed later during retrieval. But memory follows the same principle as perception in terms of active construction and reconstruction in areas of perception [11,12].

Memory can be classified into two types: 1) Declarative memory which includes episodic memory that deals with specific situations and semantic memory which deals with knowledge; and 2) Procedural memory which relates to motor skills [19]. Memory processing is simplified in the three actions of consolidation, retrieval then reconsolidation. Similar to the building of memory from the individual fragments of perception of self and environment as well as emotional reaction, memory retrieval acts by reconstructing the memory from the individual fragments stored. Once a memory is registered, it exists initially in a fragile state and without consolidation, can disappear and leave no trace of influence on neural circuits. Consolidation of memories leaves them permanently present and resistant to change. Consolidation starts initially in a cellular level which is followed by systemic consolidation which alters the cortico-hippocampal connections for declarative memories, and cerebellar-basal ganglia connections for procedural memories [19].

Memory follows frameworks for operation: When a decided mechanism of operation is applied to memory functions, it makes the memories formed never completely precise, and partially generalizable. The more specific the memory consolidation process is, the more specified the neural circuits used in it, and this after a certain limit is not efficient. The purpose of this generalization is to enables both us, and more primitive species to retrieve these memories in relevant situations and act accordingly [20].

Not all memories are generalizable. There is a usual balance between the level of generalizability and specificity which best serves the organism. Situations that induce fear are more generalizable to allow appropriate induction of fight and flight response in similar situations. It's when it's more generalizable than it should be do people experience disorders such as anxiety and post-traumatic stress disorder [20].

Complementary learning systems theory [21] is another proposed idea for how memory consolidation as well as memory retrieval function through generalization models that both retain some specification as well as allows for efficient relevant retrieval for information.

According to the theory, the hippocampus stores separate representations for individual memories through cellular consolidation. The systemic consolidation in the cortex then allows abstraction of common features from multiple memories which allows for generalization of memories during retrieval later on. They also propose that specific memories are 
differentiated through "pattern separation," and they too can be generalized during retrieval through "pattern completion" [21].

Innate dispositions and experiential adaptation in memories: Memory strength which includes its specification as well as the strength of consolidation and retrieval depends on the emotional reactivity associated with it. The more emotional affect is associated with it wither negative or positive, the stronger the consolidation and retrieval. The more neutral the stimuli, the more likely it would pass without remembrance [22].

This allows us to remember experiences most important to us and forget unimportant details. When taken to pathological levels, however, it can affect memory consolidation as well as retrieval. People suffering from social anxiety have a bias to focus more on threatening details in any social event compared to normal people [23]. Cases suffering from post-traumatic stress disorder [24] suffer from flashbacks which are involuntary repeated memory retrievals. These memories are fragmented and disorganized, not full to make a whole scene.

Subconscious memory: Evidence of subconscious memory affecting procedural memory comes from early reports by Brenda Milner regarding patient's H.M. changes after he underwent bilateral mesial temporal lobectomy and lost the ability to form declarative memories. Our understanding of memories at that time was not as comprehensive as our current understanding, so to a common observer, it seemed as if life has stopped moving forward for him because he was unable to generate memories and recall events from the time of the operation onwards. After detailed study of his case, however, it was shown that not all forms of memory were lost to him. After being given repeated motor tasks to perform, he showed improvement in motor skills, despite not being able to recall having been tasked the same task earlier [25].

Such subconscious neural processing memories don't involve procedural memories alone. Recent evidence has shown that the hippocampus is capable of retaining memories non-consciously [26]. One study has also found a non-conscious presentation of information for several seconds in brain region such as frontal cortex and occipital cortex involving in processing of working memories without conscious awareness of them [27].

Memory automaticity: Memory retrieval becomes automatic once repeated and learned. This includes procedural memory as shown in the case of H.M. [25]. It's also shown in the switch from working memory to long-term memory. This makes automaticity specific only to learned items, and not a generalizable process in memory consolidation [28].

\section{Decision making}

Decision-making and models of operation: Memories make us who we are, but the ability to decide is considered the definition of freedom. Contrary to common belief however, we are not truly free as well think we are. Our ability to decide is not a fully individually differentiated process. As humans, if we are put into the same situation, with the same background knowledge, the range of possible reactions can easily be predicted. What we view as intentional thought process is actually guided and built upon models that are designed by our genes and to a less degree modified by experience. The main guiding bias in decision making is survival advantage [29].

The processes of decision making are dependent on emotional processes that are associated with perception. It's executed through several brain regions, including the prefrontal cortex. The evidence behind the importance of such region in the ability to decide advantageously comes from a case report of an incident that occurred in 1848 in Vermont, US. The case was reported by Dr Harlow regarding the changes that occurred in Phineas Gage.

Gage was a functional respected person whose character and life drastically changed after suffering an insult to his brain. He had an accident at work that resulted in an iron rod traversing his skull [30].

Quoting Dr Harlow report: (The equilibrium or balance, so to speak, between his intellectual faculties and animal propensities, seems to have been destroyed. He is fitful, irreverent, indulging at times in the grossest profanity (which was not previously his custom), manifesting but little deference for his fellows, impatient of restraint or advice when it conflicts with his desires, at times pertinaciously obstinate, yet capricious and vacillating, devising many plans of future operation, which are no sooner arranged than they are abandoned in turn for others appearing more feasible. A child in his intellectual capacity and manifestations, he has the animal passions of a strong man. Previous to his injury, though untrained in the schools, he possessed a well-balanced mind, and was looked upon by those who knew him as a shrewd, smart business man, very energetic and persistent in executing all his plans of operation. In this regard his mind was radically changed, so decidedly that his friends and acquaintances said he was "no longer Gage") [30].

A review pertaining to the function of prefrontal cortex and its relation to the ability to feel and decide has been investigated by Damasio and his colleagues for a long time. His work comes in time after a prolonged period of neglect for that aspect of cognitive functions. Common sense stated that Emotions should be removed from logical scientific studies and everyone followed that rule [31].

One study have been done to 3-D reconstruct the remnants of Gage's skull together with the trajectory through which the rod has traversed his skull [32] the lesion he most likely have sustained was a bilateral ventromedial prefrontal cortex. Evidence from other cases with similar lesions further proves the speculations regarding the importance of such region for decision making.

They have concluded that after sustaining lesions in the ventromedial prefrontal cortex, the patient's intellect remains intact. Their memory, language and attention remain intact, as well as their motor and sensory functions. Grossly speaking, there shouldn't be anything the matter with them [31]. 
What they lack, however, is emotional reaction and as a result their behavior gets disrupted in profound ways. They don't engage emotions in social situations. They don't feel happiness when they should, jealous when they should or embarrassed when they should. As a result, their ability to decider in social situations rationally and advantageously gets profoundly compromised [32].

Out of evidence from Phineas gage and cases like him, Professor Antonio Damasio came up with the somatic marker hypothesis [1]. The hypothesis states that mental images, being visual, tactile, auditory, or any other form of thought is marked somatically with an associated emotion. This also includes thoughts we have regarding possible outcomes whenever we think of planning regarding the future. Somatic marking manifests as 'gut feeling' that allows a rapid, advantageous decision making.

To further explain, a simple example is warranted. If a child, with a still basic poor of knowledge pulls a table cover and a vase falls and hits him, the next time he would move away from the piece of cloth, or cry if put under it. This is similar to the operant conditioning learning model proposed by B.F. Skinner [33]. How the somatic hypothesis expands further is by proposing the brain regions responsible for this process of learning as well as propose an important point of view: Not all the processing is conscious. Most of the thought process we undergo to reach a decision surprisingly goes subconsciously. In normal healthy situations, our mind scans through possible actions and associated somatic markers and immediately removes those associated with possible outcome that can have negative emotions with it, i.e. result in our sadness, anger, pain... etc. It provides in the end the best options to our conscious brain and that goes for deliberate thought process.

Decision making innate systems and experiential adaptation: How we decide is built upon what we know and what we imagine can be the outcome of our actions. Once we fail to know, and more importantly, once we fail to feel. We lose our senses that guide us. We are forced then to rely on pure logic and calculations to decide. This, contrary to common belief, is not efficient, time consuming and doesn't necessarily result in wise decisions $[1,31,32]$.

The system for operation here is innate. It's generalizable to all human populations. It develops with experience and becomes individualized, but how it acts within certain situations follows generalizable laws of operation whose aim is to ensure our survival.

Experience can affect the way people make decisions. Stress is an example of an experience that influences decision processing [34]. Stress can alter how an individual calculate risk and benefit in a situation leading to more impulsive choices. When it comes to pathological levels such as in anxiety and depression, risky and irrational choices, specifically within the context of judging relative cost and benefit in conditions of conflict, are often the result [34].

Decision making and automation: The system is also automated for most of its part. The automation favors the presence of vast set of knowledge pool from which possible scenarios can be pulled. Louis Pasteur was quite wise when he said "In the fields of observation, chance favors only the prepared mind". He should have ideally put the phrase of "intuition" instead of chance to make the statement more accurate.

Several studies, including a recent one in 2019 have demonstrated the presence of an automated selection bias when it comes to options that concern reward and punishment, favoring recent reward options [35].

Decision making and subconscious process: One of the most pronounced evidence of such notion comes from a simple experiment done on a patient who suffered herpes encephalitis and after it developed a severe form of retrograde amnesia spanning his last 2 decades of life as well as a defect in declarative memory consolidation. The experiment was called 'good guy/bad guy' experiment [35]. It involved a week long exposure to 3 people, a good guy who would be very pleasant and complementary and grant him treats, a neutral one who was like a businessman, and a bad guy who always refused treating the subject, requested him to do tedious tasks and was all together in disagreeable demeanor.

Upon assessment of the result, the subject would avoid the bad guy, would not smile at him and would repeatedly choose the good guy when asked using a 2-alternative force choice questionnaire where two pictures are put in front of him and he is asked to choose a person [36].

The subject during the whole experiment was unable to recall having met any of the people in the experiment, indicating the presence of subconscious processing that guides decision making.

\section{Conclusion}

The building of any theoretic model of operation takes into consideration the limitations that are naturally associated with it. There is always a gap of knowledge that exists between any theoretical model and actual natural phenomena. As long suggested by Plato [37], science studies can never tell us the nature of the world in its reality, it can only model it to us through our observations of it.

The functions of the central nervous system, specifically those related to the 'mind' may appear very individualized and different between people. However, when seen in the grand scheme of neural processes in our species, they appear to have common characteristics and laws that govern their function.

The common characteristics proposed in this article should serve as frameworks for future studies, keeping in mind with it that our mental lives are the application of these models.

Our lives are quite automated, very structured and if known in depth, the study of it is very predictable that for a while you might actually question the true limitations of our individualism.

\section{Author Declarations}

No conflict of interest. 
No funding.

No writing assistance.

The author is sole contributor to the idea and the writing of the manuscript.

\section{References}

1. Damasio AR (1996) The somatic marker hypothesis and the possible functions of the prefrontal cortex. Philos Trans R Soc Lond B Biol Sci 351: 1413-1420.

2. Power JD, Schlaggar BL (2017) Neural plasticity across the lifespan. Wiley Interdiscip Rev Dev Biol 6.

3. Semënov MV (2019) Adult hippocampal neurogenesis is a developmental process involved in cognitive development. Front Neurosci 13: 159.

4. Jylkkä J, Railo H (2019) Consciousness as a concrete physical phenomenon. Conscious Cogn 74: 102779.

5. Lam SS, White-Schwoch T, Zecker SG, et al. (2017) Neural stability: A reflection of automaticity in reading. Neuropsychologia 103: 162-167.

6. Schwartz MF, Olufunsho F, Kim F, et al. (2012) The dorsal stream contribution to phonological retrieval in object naming. Brain 135: 3799-3814.

7. Mateos-Aparicio P, Rodríguez-Moreno A (2019) The impact of studying brain plasticity. Front Cell Neurosci 13: 66.

8. Scott HK, Cogburn M (2020) Piaget. In: StatPearls [Internet] Treasure Island (FL): StatPearls Publishing.

9. Stoltz T (2018) Consciousness in piaget: Possibilities of understanding. Psicologia: Reflexão e Crítica 31: 30.

10. Gupta M, Bordoni B (2020) Neuroanatomy, visual pathway. In: StatPearls [Internet]. Treasure Island (FL): StatPearls Publishing.

11. Dijkstra N, Mostert P, Lange FP, et al. (2018) Differential temporal dynamics during visual imagery and perception. Elife 7: e33904.

12. Dijkstra N, Zeidman P, Ondobaka S, et al. (2017) Distinct topdown and bottom-up brain connectivity during visual perception and imagery. Sci Rep 7: 5677.

13. Adelson EH (1993) Perceptual organization and the judgment of brightness. Science 262: 2042-2044.

14. Liu TT, Behrmann M (2017) Functional outcomes following lesions in visual cortex: Implications for plasticity of high-level vision. Neuropsychologia 105: 197-214.

15. Sacks O (2010) The mind's eye. Print.

16. Ionescu MR (2016) Subliminal perception of complex visual stimuli. Rom J Ophthalmol 60: 226-230.

17. Meneguzzo P, Tsakiris M, Schioth HB, et al. (2014) Subliminal versus supraliminal stimuli activate neural responses in anterior cingulate cortex, fusiform gyrus and insula: A meta-analysis of fMRI studies. BMC Psychol 2: 52.
18. Teachman BA, Joormann J, Steinman SA, et al. (2012) Automaticity in anxiety disorders and major depressive disorder. Clin Psychol Rev 32: 575-603.

19. Bisaz R, Travaglia A, Alberini CM (2014) The neurobiological bases of memory formation: From physiological conditions to psychopathology. Psychopathology 47: 347-356.

20. Xu W, Südhof TC (2013) A neural circuit for memory specificity and generalization. Science 339: 1290-1295.

21. Nakashiba T, Cushman JD, Pelkey KA, et al. (2012) Young dentate granule cells mediate pattern separation, whereas old granule cells facilitate pattern completion. Cell 149: 188-201.

22. Sun Q, Gu S, Yang J (2018) Context and time matter: Effects of emotion and motivation on episodic memory overtime. Neural Plasticity.

23. Morgan J (2010) Autobiographical memory biases in social anxiety. Clinical Psychology Review 30: 288-297.

24. Rybak-Korneluk A, Wichowicz HM, Żuk K, et al. (2016) Autobiographical memory and its meaning in selected mental disorders. Psychiatr Pol 50: 959-972.

25. Milner B (1962) Les troubles de la memoire accompagnant des lesions hippocampiques bilaterales. In: Physiologic de l'Hippocampe. Paris: Centre National de la Recherche Scientifique, 257-272.

26. Duss SB, Reber TP, Hänggi J, et al. (2014) Unconscious relational encoding depends on hippocampus. Brain 137: 3355-3370.

27. Bergström F, Eriksson J (2018) Neural evidence for non-conscious working memory. Cereb Cortex 28: 3217-3228.

28. Servant M, Cassey P, Woodman GF, et al. (2018) Neural bases of automaticity. J Exp Psychol Learn Mem Cogn 44: 440-464.

29. Damasio A (1999) The feeling of what happens: Body, emotion and the making of consciousness. Harcourt College Publishers.

30. Harlow JM (1993) Recovery from the passage of an iron bar through the head. Hist Psychiatry 4: 274-281.

31. Bechara A, Damasio H, Damasio AR (2000) Emotion, decision making and the orbitofrontal cortex. Cereb Cortex 10: 295-307.

32. Damasio H, Grabowski T, Frank R, et al. (1994) The return of phineas gage: Clues about the brain from the skull of a famous patient. Science 264: 1102-1105.

33. Isai Amutan Krishnan (2014) A review of BF skinner's 'Reinforcement Theory of Motivation'. Journal of Research in Education Methodology, 5.

34. Friedman A, Homma D, Bloem B, et al. (2017) Chronic stress alters striosome-circuit dynamics, leading to aberrant decision-making. Cell 171: 1191-1205.

35. Zhao WJ, Diederich A, Trueblood JS, et al. (2019) Automatic biases in intertemporal choice. Psychon Bull Rev 26: 661-668.

36. Damasio A, Damasio H, Tranel D (2013) Persistence of feelings and sentience after bilateral damage of the insula. Cereb Cortex 23: 833-846.

37. Asano Kozi (1993) Degrees of reality in plato: Part I. Aichi 10: 1-14.

DOI: $10.36959 / 447 / 349$

Copyright: (C) 2020 Shangab MOM. This is an open-access article distributed under the terms of the Creative Commons Attribution License, which permits unrestricted use, distribution, and reproduction in any medium, provided the original author and source are credited. 